Pacific Journal of Mathematics

ALGEBRAIC CHARACTERIZATION OF THE VACUUM FOR
QUANTIZED FIELDS TRANSFORMING NONUNITARILY 
Pacific Journal of Mathematics

ALGEBRAIC CHARACTERIZATION OF THE VACUUM FOR
QUANTIZED FIELDS TRANSFORMING NONUNITARILY 


\title{
ALGEBRAIC CHARACTERIZATION \\ OF THE VACUUM FOR QUANTIZED FIELDS TRANSFORMING NON-UNITARILY
}

IRVING SEGAL

Dedicated to the memory of Henry A. Dye

\begin{abstract}
The vacuum as an expectation value form on the Clifford or Weyl algebra over an orthogonal or symplectic real linear space, invariant under a given group of automorphisms of such, is treated without assumptions as to self-adjointness or positivity. This is necessary for the quantization of fields that transform non-unitarily, in particular indecomposably, such as the full section spaces of typical conformally invariant bundles over space-times. A stability condition in the nature of positivity of the energy is shown to be sufficient to characterize the vacuum for the basic case of a one-parameter group. In application e.g. to spannor fields transforming under $S U(2,2)$, this results in a vacuum invariant under the maximal subgroup $K$, giving rise to a natural broken symmetry.
\end{abstract}

1. Introduction. From early heuristic beginnings, quantization has developed into a vast enterprise that is in large part rigorously mathematical. The finite-dimensional case in standard quantum mechanics was settled essentially by Stone and van Neumann, whose work paid important mathematical dividends. The infinite-dimensional case was more refractory, but there are now simple and natural characterizations of the free boson and fermion quantum fields. At first the quantization procedure treated only systems whose single-particle structure transformed unitarily under the basic symmetry group, as in the most familiar physical cases (e.g. the Klein-Gordon, Maxwell, and Dirac equations). The associated unitary action on the quantized field Hilbert space was then essentially the direct sum of the symmetrized or skew-symmetrized tensor powers of the single-particle representation, depending on the "statistics" of the field, i.e. whether bosons or fermions were involved.

In practice, the single-particle action was often, in the more interesting cases, such as when interactions were involved, not unitary or at least not manifestly unitary, but only symplectic in the case of bosons or orthogonal in the case of fermions. This led to the study of the question on unitary implementability on the quantum field of 
such single-particle actions, and in particular to the treatment of the infinite-dimensional harmonic and spin representations, of the infinite symplectic and orthogonal groups respectively. There was a definitive answer, which showed that unitary implementability was limited to those symplectics or orthogonals that were close to being unitary or at least unitarizable.

This excluded many transformations that occurred in practice, as so-called "canonical" transformations of quantized fields. In order to accommodate these, it was necessary to give ground on the spatial implementation and adjust to a $C^{*}$-algebraic treatment, in which states were no longer represented by vectors in a priori Hilbert space, but by normalized positive linear functionals on the algebra. At the same time, there was an a posteriori Hilbert space, determined in part by symmetry considerations, but not at all uniquely, particularly in the infinite dimensional case. This a posteriori, or "physical" quantum field Hilbert space, was determined in part by stability considerations, usually expressed by the phrase "positivity of the energy", but in general the energy itself is an a posteriori concept.

This matter of stability has not yet received much attention in the finite-dimensional case, which has otherwise been effectively explored in the past several decades in connection with the representation theory of semisimple and other Lie groups. In view of the relatively wide familiarity with this context, it may clarify the present concern with the infinite-dimensional case by comparing it with the better known finite-dimensional context. The finite-dimensional harmonic oscillator is uniquely determined by its invariance under the unitary group. Specifically, if $\mathbf{H}$ is a finite-dimensional complex Hilbert space, and if $(\mathbf{K}, W)$ is a Weyl system over $H$ in the sense that $W$ is a continuous map from $\mathbf{H}$ to operators on the complex Hilbert space $\mathbf{K}$ such that $W(z) W\left(z^{\prime}\right)=e^{(i / 2) \operatorname{Im}\left\langle z, z^{\prime}\right\rangle} W\left(z+z^{\prime}\right)$ for arbitrary $z$ and $z^{\prime}$ in $\mathbf{H}$, there is then a unique (trace-class) state $E$ of the $W^{*}$-algebra generated by the $W(z)$ that is invariant under the induced action of the unitary transformations on $\mathbf{H}$. This induced action $\Gamma(U)$ of the unitary $U$ on $\mathbf{H}$ is the unitary operator $V$ on $\mathbf{K}$, unique within a constant phase factor, such that $V W(z) V^{-1}=W(V z)$ for all $z$. This state $E$ is roughly the ground-state of the harmonic oscillator. In the infinitedimensional case, the formalism is well-defined, and in particular the continuous mapping $\Gamma$ from unitaries on $\mathbf{H}$ to those on $\mathbf{K}$ exists, but the state $E$ invariant under all $\Gamma(U)$ is not at all unique [9]. It becomes unique and represents the conventional vacuum in this context only 
by virtue of a stability constraint. Indeed, the constraint of "positivity of the energy" is itself strong enough to imply unicity, without any assumption of invariance, except under the one-parameter group whose generator represents the energy.

In all of this work there is a so-called "single-particle" representation of the fundamental symmetry group that is irreducible, or a direct sum or direct integral of irreducibles. Recent natural particle models have however interlocked the elementary particles in a way represented mathematically by indecomposability of the underlying group representation $[8,9 A, 11]$. The physical processes of partical decays and weak interactions are potentially quite economically described in terms of indecomposable representations, and the section spaces of induced bundles over space-times, which appear to represent the natural habitat of classical fields, are often indecomposable. But the indecomposability produces serious and unprecedented problems of quantization, i.e. the appropriate replacement of the numericallyvalued components of fields by operator-valued components.

From a mathematical position, the key problem may be described as the absence of a canonical or a priori adjunction operator ${ }^{*}$ for the algebra of field observables; rather the ${ }^{*}$ must be solved for. The basis of the solution must be a combination of invariance and stability constraints, for the reasons indicated. In general terms, the context is algebraic, without being $C^{*}$-algebraic on an a priori basis, as in Haagerup [6], but more structured than in that work, by virtue of the cited constraints. The treatment here will be purely mathematical, apart from some motivational comments, but some background in algebraic quantum mechanics will be relevant (cf. e.g. Emch [4], Varadhan [12]). The $C^{*}$-algebraic analysis of physical issues involving Dirac articles is treated comprehensively by Bongaarts [3].

Henry Dye made some of the most fundamental contributions to operator algebra and its applications, especially to the modelling of theoretical physical systems. This is an offering in honor of his originality and depth that is in the same technical and philosophic vein, albeit concerned with particle rather than ergodic theory. As Henry was a man of exceptional culture and scientific generosity, this should be appropriate.

2. Fermion algebra. The need for an a posteriori determination of the * is more evident in the fermion than in the boson case, and I begin with that. However, in either case, the general mathematical context is that one is given a real linear topological space $L$; a non-degenerate 
continuous bilinear form that is either symmetric (denoted as $S$ ) or antisymmetric (denoted as $A$ ), depending on whether fermions or bosons are involved. In addition, one is given a representation $U$ of a given group $G$ into the orthogonal group $\mathrm{O}(\mathbf{L}, S)$ or the symplectic group $\operatorname{Sp}(\mathbf{L}, A)$. The basic algebra in question, or "field algebra", is the Clifford algebra $\mathbf{C}(\mathbf{L}, S)$ in the fermion case. This is generated by 1 and $\mathbf{L}$ with the relations $x y+y x=S(x, y)$ for arbitrary $x$ and $y$ in $\mathbf{L}$. It is clear that if $S$ is indefinite, $\mathbf{L}$ cannot map exclusively into either selfadjoint or skew-adjoint operators in a Hilbert space representation of $\mathbf{C}(\mathbf{L}, S)$, and indeed there is no a priori determination of $x^{*}$ for $x$ in $\mathbf{L}$.

For any $g \in G$ there is an automorphism $\gamma(g)$ of $\mathbf{C}(\mathbf{L}, S)$ (denoted simply as $\mathbf{C}$ when $\mathbf{L}$ and $S$ are clear from the context) that carries $x$ into $U(g) x$, for all $x \in \mathbf{L}$. Physical states are represented in the now familiar way by linear functionals on $\mathbf{C}$ having properties appropriate to the expectation value form for the state. These are usually normalization: $E(1)=1$, and positivity: $E\left(A^{*} A\right) \geq 0$, but without $a^{*}$ the latter important constraint cannot be directly imposed. But invariance and stability provide further constraints, and my aim here is to show that these provide a natural and effective substitute; in particular, eventually $\mathrm{a}^{*}$ is attained.

The method derives from the approach to the characterization of the vacuum developed by Segal [9] and Weinless [13]. The basic case is that in which $G$ is a one-parameter group whose generator represents an inherently non-negative quantity, such as the "energy" or "total particle number". This suffices in particular for the treatment of "chronometric" particle theory, which in mathematical form is initiated in [8]; the group $G$ is essentially $\mathrm{SU}(2,2)$, but there is a "broken symmetry", which reduces the invariance of the vacuum to the maximal compact subgroup $K$ (which is also maximal). $K$ includes a central one-parameter subgroup, whose generator may be interpreted as the energy, and in terms of which the vacuum may be defined and treated.

3. Some technical preliminaries. I recall here some terminology and results that will serve also to fix notation.

A topological linear space $\mathbf{L}$ over the real or complex field will be called Hilbertizable if it is isomorphic to a Hilbert space over the same field (linearly/bicontinuously). An orthogonal topological linear space $(\mathbf{L}, S)$ includes a non-degenerate bilinear form $S$ over $\mathbf{L}$. It is Hilbertizable as such if $\mathbf{L}$ is isomorphic to a Hilbert space in such a way that $S$ is represented by a bounded invertible operator, i.e. takes the 
form $S(x, y)=\langle T x, y\rangle$, where $T$ is invertible. A symplectic topological linear space is defined similarly; such a space $(\mathbf{L}, A)$ may alternatively be defined as one which within isomorphism is a complex Hilbert space regarded as a real topological space, with the form $A$ consisting of $\operatorname{Im}\langle\cdot, \cdot\rangle$.

A covariant quantization of a structure $(\mathbf{L}, F, G, U)$, where $\mathbf{L}$ is a real linear topological space on which $F$ is a continuous bilinear form, $G$ a given topological group, and $U$ a continuous representation of $G$ on $\mathbf{L}$ that leaves $F$ fixed, will be defined here only in the cases in which $F$ is either symmetric or antisymmetric. When $F$ is symmetric, it is defined to consist of a structure $(\mathbf{K}, \Phi, \Gamma, v)$, where $\mathbf{K}$ is a complex Hilbert space; $\Phi$ is a real-linear map from $\mathbf{L}$ into bounded linear operators on $\mathbf{K}$ satisfying the canonical relations ('ACR's for short):

$$
\Phi(x) \Phi(y)+\Phi(y) \Phi(x)=F(x, y) ;
$$

$\Gamma$ is a continuous unitary representation of $G$ on $\mathbf{K}$ such that

$$
\Gamma(g) \Phi(x) \Gamma(g)^{-1}=\Phi(U(g) x) ;
$$

and $v$ is a unit vector in $\mathbf{K}$ that is cyclic for the $\Phi(x), x \in \mathbf{L}$, and left fixed by all $\Gamma(g)$. (In these relations, $x$ and $y$ are arbitrary in $\mathbf{L}$, and $g$ is arbitrary in $G$.) Such a quantization is stable if $G$ is a Lie group in the Lie algebra of which is given a convex invariant cone $\mathbf{P}$ (relative to which stability is defined), such that for all $X \in \mathbf{P}$, the self-adjoint generator of the one-parameter unitary group $\Gamma\left(e^{t X}\right)$ is non-negative.

If $L$ is a complex Hilbert space $H$ regarded as a real space, with $F=\operatorname{Re}\langle\cdot, \cdot\rangle, G=R^{1}$ as an additive group, and $U$ a one-parameter unitary group on $\mathbf{H}$ with positive self-adjoint generator, then it is known that the structure $\mathbf{F}$ (for Fermi) $=(\mathbf{K}, \Phi, \Gamma, v)$ defining a stable covariant quantization exists, and is unique within unitary equivalence. Moreover, this quantization has the property that the representation $\Gamma$ extends in the following manner. There is a continuous representation $\Gamma^{\prime}$ of the unitary group on $\mathbf{H}$ by unitary operators on $\mathbf{K}$ such that $\Gamma^{\prime}(U(g))=\Gamma(g)$ for all $g \in G$, and which moreover has the property that the self-adjoint generator of $\Gamma^{\prime}(V(t))$ is non-negative for all continuous one-parameter groups $V(\cdot)$ on $\mathbf{H}$ of non-negative generator. Thus stability with respect to one one-parameter unitary group with positive generator implies stability with respect to all such groups. The resulting universal structure $\left(\mathbf{K}, \Phi, \Gamma^{\prime}, v\right)$ is called the free fermion field over $\mathbf{H}$; $v$ is called the vacuum vector. In the future the prime on $\Gamma^{\prime}$ will be dropped, the context sufficing to distinguish between this representation and the related one $\Gamma$. 
When $F$ is antisymmetric, the definitions are parallel, except for the intervention of unbounded operators. The commutation relation

$$
\boldsymbol{\Phi}(x) \Phi(y)-\boldsymbol{\Phi}(y) \Phi(x)=i A(x, y)
$$

must be interpreted as an inclusion between the operator on the left and that on the right, that on the left being only densely defined, and it is technically advantageous (and necessary to avoid irrelevant pathology) to use instead the Weyl relations. A Weyl system over $(\mathbf{L}, A)$ is a structure $(\mathbf{K}, W)$ in which $\mathbf{K}$ is a complex Hilbert space and $W$ is a continuous map from $\mathbf{L}$ to the unitary operators on $\mathbf{K}$ that satisfies the relations: $W(z) W\left(z^{\prime}\right)=e^{\frac{1}{2} i A\left(z, z^{\prime}\right)} W\left(z+z^{\prime}\right)$. In terms of $W, \Phi$ is defined as follows: $\Phi(x)$ is the self-adjoint generator of the one-parameter unitary group $W(t x), t \in R^{1}$. If $\mathbf{H}$ is a given complex Hilbert space, there is a universal free boson field with properties parallel to those in the fermion case, and denoted in a similar way. In these terms the free boson and fermion vacua may be characterized as follows (Segal [9], Weinless [13]).

Let $(L, F)$ be a given Hilbertizable symplectic or orthogonal space, and let $U(\cdot)$ be a given continuous one-parameter group of automorphisms of $(L, F)$ leaving no nonzero vector fixed. Then there exists a free quantization for $\left(L, F, R^{1}, U\right)$ if and only if $U(\cdot)$ is unitarizable with a positive self-adjoint generator, where unitarization includes the mapping of $F$ into the imaginary or real part of the complex Hilbert structure. Moreover, the unitarization and the free quantization is then unique, within unitary equivalence.

As noted earlier,the condition that the self-adjoint generator of the group $\Gamma(t)$ be non-negative is most essential; but in the case of a oneparameter group of automorphisms $\gamma(t)$ induced by the action of such a group on an invariant algebra, there is no a priori equivalent condition. When the algebra is a $C^{*}$-algebra, and there is an associated invariant state $E$, then the canonical representation associated with $E$ may be used to define the spatial group $\Gamma(t)$; but the Clifford algebra associated with an indefinite symmetric form is not canonically $a^{*}$-algebra at all. Apart from the positivity of the linear functional associated with the state vector $v$, the stability condition, i.e. the non-negativity of the generator of the group $\Gamma(\cdot)$, may be stated as follows: For all elements $P$ and $Q$ of the invariant algebra under the $\gamma(t)$, the function of $t, E(\gamma(t)(P) Q)$ is devoid of negative-frequency components. I show here that this condition alone, without any assumption of positivity, apart from the normalization $E(1)=1$, is sufficient to 
characterize the vacuum of the putative quantized field. Moreover, as earlier, the vacuum exists if and only if the representation $U(\cdot)$ is appropriately unitarizable.

4. The fermionic vacuum. In the simplest cases such as that of the Dirac electron, a stable unitary representation of a group on a complex Hilbert space is substantially given. In this case the Poincaré group acts unitarily on the Hilbert space of normalizable wave functions, in an entirely explicit way (cf. Bargmann and Wigner [1]; Mackey [7]). In more complex cases, much less is given. Consider for example the tensor product of the action of the group $G=\operatorname{SU}(2,2)$ on the section space of the scalar bundle of weight 2 over the conformal compactification of Minkowski space with the defining representation $R$ of $G$, which forms an interesting model known as the spannors (cf. Paneitz et al. [8]). The action $V$ on the scalar sections is unitary and decomposes as a direct sum $V=V^{+} \oplus V^{0} \oplus V^{-}$, respectively of positive, mixed, and negative energy. The component $V^{+} \times R$ of the tensor product has a 3-step composition series whose factors are all stably unitarizable; but the representation as a whole is indecomposable. This poses not merely a technical challenge, but may serve to describe observed decay processes more effectively than the nonlinear Lagrangians that have been the exclusive model for interactions in conventional relativistic theory.

This section treats the general issue of how to deal with this type of situation, by means that will yield concrete results for cases such as that indicated. The ideas of stability and broken symmetry will be seen to be effective, in conjunction with symmetry.

Let the group $G$ act on the Hilbertizable real orthogonal space $(\mathbf{L}, S)$ by the representation $U(\cdot)$. The associated fermion field is represented algebraically by the Clifford algebra $\mathbf{C}$ over $(\mathbf{L}, S)$, on which $G$ acts canonically as a group of automorphisms, but there is no given * on C. The basic case is that in which $G$ is a one-parameter group. The Clifford algebra here will be understood throughout as that with complex coefficients, irrespective of whether the underlying linear spacecum-quadratic form is real or complex.

THEOREM 1. Let $U(\cdot)$ be a continuous one-parameter group of automorphisms of the Hilbertizable (real) orthogonal space $(\mathbf{L}, S)$ that leaves no vector in $\mathbf{L}$ fixed, other than 0 . Let $\mathbf{C}$ denote the Clifford algebra over $(\mathbf{L}, S)$, and let $u(\cdot)$ denote the one-parameter group of 
automorphisms of $\mathbf{C}$ canonically induced from $U(\cdot)$. Suppose there exists a linear functional $\mathbf{E}$ on $\mathbf{C}$ with the following properties (where $u(t)(Q)$ is denoted as $Q^{t}$, for $\left.Q \in \mathbf{C}\right):$ (i) $E(1)=1$; (ii) $E$ is $u(\cdot)$ invariant: $E\left(Q^{t}\right)=E(Q)$ for arbitrary $Q \in \mathbf{C}$; (iii) for arbitrary $Q$ and $R$ in $\mathbf{C}, E\left(Q^{t} R\right)$ is as a function of the boundary values of a bounded analytic function in the upper half-plane; (iv) $E\left(x_{1} x_{2} \cdots x_{r}\right)$ is a jointly continuous function of $x_{1}, x_{2}, \ldots, x_{r}\left(x_{j} \in \mathbf{L}\right) ;(\mathbf{v})$ if $\mathbf{L}_{+}$and $\mathbf{L}_{-}$are $U(\cdot)$-invariant linear submanifolds of $\mathbf{L}$ on which $S$ is respectively positive and negative definite, then $\bar{E}\left(x_{1} x_{2} \cdots x_{r}\right)=(-1)^{r} E\left(x_{r} \cdots x_{2} x_{1}\right)$, if the $x_{j}$ are either in $\mathbf{L}_{+}$or $\mathbf{L}_{-}$, and $r$ is the number of $x_{j}$ in $\mathbf{L}_{-}$.

Then there exists a complex Hilbert structure on $\mathbf{L}$, compatible with its given real Hilbertizable structure, and a projection $P$ on $\mathbf{L}$ as a complex Hilbert space, denoted $\mathbf{H}$, such that:

(1) $U(\cdot)$ is unitarizable with positive self-adjoint generator on $\mathbf{H}$;

(2) $S(x, y)=\operatorname{Re}\langle(2 P-1) x, y\rangle$;

(3) $E$ coincides with the free fermionic vacuum over $\mathbf{H}$.

Proof. Applying $E$ to both sides of the equation $x^{t} y+y x^{t}=S\left(x^{t}, y\right)$, it follows that $E\left(x^{t} y\right)+E\left(y x^{t}\right)=S\left(x^{t} y\right)$. Now $E\left(x^{t}, y\right)$ is bounded as a function of $t$ for arbitrary fixed $x$ and $y$, and $E\left(y x^{t}\right)=E\left(y^{-t} x\right)$, which is similarly bounded. Thus $S(U(t) x, y)$ is bounded as a function of $t$ for arbitrary $x$ and $y$.

Now choose an arbitrary real orthogonal structure on $\mathbf{L}$ that is representative of its given Hilbertizable structure, with real inner product $((\cdot, \cdot))$. Relative to this structure, $S$ takes the form $S(x, y)=((B x, y))$, where $B$ is a bounded invertible self-adjoint operator. In particular, $S(U(t) x, y)=((B U(t) x, y))$, from which it follows by the uniform boundedness principle that $\|B U(t)\|$ is bounded as a function of $t$. Hence $\|U(t)\|$ also is bounded. Now let $\mathbf{T}$ denote a translationinvariant generalized mean for bounded functions on $R^{1}$, arbitrary but fixed, and let $Q(x, y)=\mathbf{T}((U(\cdot) x, U(\cdot) y))$. Then $Q$ is a $U(\cdot)$-invariant positive definite form on $\mathbf{L}$ that is topologically equivalent to $((\cdot, \cdot))$. Consequently there exists a bounded linear operator $R$ on $\mathbf{L}$ that is self-adjoint relative to $Q$, and such that $S(x, y)=Q(R x, y)$ for arbitrary $x$ and $y$ in L. $R$ is invertible by virtue of the Hilbertizability of $(\mathbf{L}, S)$, and the unicity of $R$ implies that it commutes with all $U(t)$.

From the $Q$-self-adjointness of $R$ it follows that $R=R_{+}-R_{-}$, where the $R_{ \pm}$are non-negative $Q$-self-adjoint operators with orthogonal ranges $\mathbf{L}_{ \pm}$, relative to which they are respectively invertible. Now setting $|S|(x, y)=Q(|R| x, y)$ (where $|R|=R_{+}+R_{-}$), then $|S|$ is a 
positive-definite symmetric form on $\mathbf{L}$ that is representative of the given Hilbertizable structure, invariant under all $U(t)$, and such that $S(x, y)=|S|(K x, y)$, with $K=\operatorname{sgn} R=R|R|^{-1}$.

To construct the complex structure, note the

LEMMA. Let $V$ be a continuous one-parameter group of orthogonal transformations on the real Hilbert space $\mathbf{N}$ leaving no nonzero vector fixed. Then there exists a unique complex Hilbert space structure on $\mathbf{N}$ relative to which $V$ is unitary and has a positive self-adjoint generator.

Proof of lemma. $V$ has the spectral resolution

$$
V(t)=\int_{-\infty}^{\infty}\left(\begin{array}{cc}
\cos t \lambda & -\sin t \lambda \\
\sin t \lambda & \cos t \lambda
\end{array}\right) d E(\lambda)
$$

where $E(\cdot)$ is a resolution of the identity. Since no nonzero vector is fixed by all the $V(t), 0$ is not in the point spectrum for $E(\cdot)$. For negative values of $\lambda$ the integrand is equivalent to that for $|\lambda|$, via a similarity transformation on the underlying 2-dimensional space. Accordingly, by a corresponding redefinition of $E(\cdot)$, the integration range may be restricted to the interval $(0, \infty)$. Now setting $J=$ $\int_{0}^{\infty}\left(\begin{array}{cc}0 & -1 \\ 1 & 0\end{array}\right) d E(\lambda), J$ is a complex structure that is orthogonal and commutes with the $V(t)$. If $P$ is the given real inner product, let $\langle x, y\rangle=$ $P(x, y)+i P(x, J y)$; then the $V(t)$ are unitary relative to $\langle\cdot, \cdot\rangle$, having the spectral resolution $V(t)=\int e^{i t \lambda} d E(\lambda)$. The self-adjoint generator of $V(\cdot)$ relative to the complex Hilbert structure on $\mathbf{N}$ is $\int \lambda d E(\lambda)$, and so positive. The unicity of $J$ follows from Lemma 1.6 of Weinless [13].

Completion of proof of theorem Let $J_{ \pm}$be the complex structures on $\mathbf{L}_{ \pm}$given by the lemma, with $\mathbf{N}$ replaced by $\mathbf{L}_{ \pm}, V$ by $U$, and the orthogonal structures defined by $S \mid \mathbf{L}_{+}$and $-S \mid \mathbf{L}_{-}$. Setting $J=$ $J_{+} \oplus J_{-}$, then $J$ is a complex structure on $\mathbf{L}$ that commutes with the $U(t)$, is orthogonal with respect to both $S$ and $|S|$. Since the $U_{ \pm}$each have a positive self-adjoint generator, so also does $U$. Now combining the positive definite form $|S|$ with the complex structure $J$, a unique complex Hilbert structure $\mathbf{H}$, whose real linear space is $\mathbf{L}$ is obtained, for which $|S|$ is the real part of the inner product.

Now let $\mathbf{L}^{c}$ denote the complexification of $\mathbf{L}$, consisting of all $x+i y$ with $x, y \in \mathbf{L}$ (here $i$ is a coefficient in the Clifford algebra, and distinct from $J$ ). For any symmetric bilinear form $F$ on $\mathbf{L}$, let $F^{c}$ denote the corresponding complex symmetric bilinear form on $\mathbf{L}^{c}$ :

$$
F^{c}\left(x+i y, x^{\prime}+i y^{\prime}\right)=F\left(x, x^{\prime}\right)-F\left(y, y^{\prime}\right)+i\left(F\left(x, y^{\prime}\right)+F\left(x^{\prime}, y\right)\right) \text {. }
$$


$\mathrm{O}(\mathbf{L}, F)$ is mapped isomorphically into the complex orthogonal group $\mathrm{O}\left(\mathbf{L}^{c}, F^{c}\right)$ by the map $U \rightarrow U^{\prime}$, where $U^{\prime}(x+i y)=U x+i U y$. The complex Clifford algebra over $\left(\mathbf{L}^{c}, F^{c}\right)$ is identical as an algebra to $\mathbf{C}(\mathbf{L}, F)$, and the respective actions of $\mathrm{O}\left(\mathbf{L}^{c}, F^{c}\right)$ and of $\mathrm{O}(\mathbf{L}, F)$ on these algebras are intertwined by the indicated map of $\mathrm{O}(\mathbf{L}, F)$ into $\mathrm{O}\left(\mathbf{L}^{c}, F^{c}\right)$.

Applying this to the cases $F=S$ and $F=|S|$, the resulting forms $S^{c}$ and $|S|^{c}$ are equivalent as complex symmetric bilinear forms on $\mathbf{L}^{c}$, via the complex linear map $W:\left(x^{+}+x^{-}\right)+i\left(y^{+}+y^{-}\right) \rightarrow\left(x^{+}-y^{-}\right)+$ $i\left(y^{+}+x^{-}\right)$, where the superscripts indicate the respective components in $\mathbf{L}^{c}$. For this means that $C^{c}\left(z, z^{\prime}\right)=|S|^{c}\left(W z, W z^{\prime}\right)$ for arbitrary $z$ and $z^{\prime}$ in $\mathbf{L}^{c}$. This is straightforward to verify by consideration of the separate cases $z$ or $i z$ in $\mathbf{L}_{ \pm}$, and similarly for $z^{\prime}$. The Clifford algebras $\mathbf{C}\left(\mathbf{L}^{c}, \boldsymbol{F}^{c}\right)$ and $\mathbf{C}\left(\mathbf{L}^{c},|S|^{c}\right)$ are thus canonically identifiable.

The real linear subspace $\mathbf{L}_{+}+i L_{-}$of $\mathbf{L}^{c}$, as a complex Hilbert space with real part of its inner product equal to $S^{c} \mid \mathbf{L}_{+}+i \mathbf{L}_{-}$, and the complex structure $J_{+}$on $\mathbf{L}_{+}$and $J_{-}$extended by commutativity with $i$ on $\mathbf{L}_{-}$, is isomorphic to $\mathbf{H}$ via the mapping from $x^{+}+x^{-}$to $x^{+}+i x^{-}$. On $\mathbf{C}(\mathbf{H})$ there is a natural adjunction operation * uniquely determined by the condition that the elements of $\mathbf{H}$ are self-adjoint; and by the preceding observation, $\mathbf{C}(\mathbf{H})$ is identifiable with $\mathbf{C}(\mathbf{L}, S)$. With respect to the ${ }^{*}$ in $\mathbf{C}(\mathbf{H}), E\left(u^{*}\right)=\bar{E}(u)$ for arbitrary $u \in \mathbf{C}$. To establish this it suffices by linearity to consider the case when $u=y_{1} y_{2} \cdots y_{n}$, where the $y_{j}$ are either in $\mathbf{L}_{+}$or $\mathbf{L}_{-}$. Now $y^{*}= \pm y$ according as $u \in \mathbf{L}_{ \pm}$, so that $E\left(u^{*}\right)=(-1)^{r} E\left(y_{n} \cdots y_{2} y_{1}\right)$, where $r$ is the number of $y_{j}$ that are in $\mathbf{L}_{-}$; and by hypothesis on $E$ this is the same as $\bar{E}\left(y_{1} y_{2} \cdots y_{n}\right)$.

To show that $E$ coincides with the vacuum of the free fermion field over $\mathbf{H}$, it therefore suffices to establish

Lemma 2. Let $\mathbf{H}$ be a complex Hilbert space, let $U(\cdot)$ be a oneparameter unitary group on $\mathbf{H}$ having positive self-adjoint generator, and let $E$ be a self-adjoint linear functional on $\mathbf{C}(\mathbf{H})$ with the property that $E\left(Q^{t} R\right)$ is as a function of the boundary values of a bounded analytic function in the upper half-plane, for arbitrary $Q, R \in \mathbf{C}(\mathbf{H})$. Then $E$ is the vacuum expectation value functional of the free fermion field over $\mathbf{H}$.

Proof of lemma. For arbitrary $x \in \mathbf{L}, E(x)$ is a continuous linear functional of $x$, and hence of the form $E(x)=\langle x, y\rangle$, where $y$ is a unique vector in L. On the other hand, $E\left(x^{t}\right)=E(x)$, which by the 
unicity of $y$ implies that $U(-t) t=y$ for all $t$. But $U(\cdot)$ leaves no nonzero vector fixed, i.e. $y=0$ and $E(x)=0$ for all $x \in \mathbf{L}$.

This is the first step of an induction argument, of which the second is logically superfluous, but clarifies the general step. Applying $E$ to both sides of the equation $x^{t} y+y x^{t}=\operatorname{Re}\langle U(t) x, y\rangle$, it follows from the self-adjointness of $E$ that $\operatorname{Re} E\left(x^{t} y\right)=\frac{1}{2} \operatorname{Re}\langle U(t) x, y\rangle$. Both the left and right sides of the last equality are the real parts of the boundary values of a bounded analytic function in the upper half-plane, as functions of $t$. Accordingly, as functions of $t$ they differ only by a constant as regards their $t$-dependence. But the vacuum of the free fermion field satisfies the hypothesized conditions also. Denoting this vacuum functional on $\mathbf{C}(\mathbf{H})$ as $E^{\prime}$, the difference $=E\left(x^{t} y\right)-E^{\prime}\left(x^{t} y\right)$ is accordingly independent of $t$, but for each $y$ a continuous linear functional of $x$, which by the argument above can be invariant under the $U(t)$ only if it vanishes.

Now as the basis of an induction hypothesis, assume that

$$
E\left(x_{1} x_{2} \cdots x_{m}\right)=E^{\prime}\left(x_{1} x_{2} \cdots x_{m}\right) \quad \text { for } m \leq n .
$$

Let $y$ and $x_{1}, x_{2}, \ldots, x_{n}$ be arbitrary in $\mathbf{H}$, and consider

$$
D=E\left(y^{t} x_{1} x_{2} \cdots x_{n}\right)-(-1)^{n} E\left(x_{n} \cdots x_{2} x_{1} y^{t}\right) ;
$$

by the Clifford relations, $y^{t} x_{1} x_{2} \cdots x_{n}-(-1)^{n} x_{n} \cdots x_{2} x_{1} y^{t}$ is an element of $\mathbf{C}(\mathbf{H})$ whose $E$ and $E^{\prime}$ expectation values agree by virtue of the induction hypothesis. But the indicated expression is of the form $E(u) \pm E\left(u^{*}\right)$, showing that either its real or its imaginary part is determined by the induction hypothesis, and so the same as that for $E^{\prime}$. By analyticity, $E\left(y^{t} x_{1} x_{2} \cdots x_{n}\right)$ and $E^{\prime}\left(y^{t} x_{1} x_{2} \cdots x_{n}\right)$ as functions of $t$ can differ only by a constant, but again a continuous linear invariant functional of $y$, so they are identical, in particular for $t=0$, completing the induction.

REMARK. If $E$ was known a priori to be a positive linear functional relative to the ${ }^{*}$, the lemma would follow from [13], Theorem 2.1, via the formation of the canonical Hilbert space representation of $\mathbf{C}(\mathbf{H})$ associated with $E$, but this follows only a posteriori. However, the induction argument given there carries over to the present case.

EXAMPLE. Let $G$ denote the group $\operatorname{SU}(1,1)$, acting in the usual way by linear fractional transformations on $S^{1}$. Let $V$ denote the unitarized action of $G$ on the space $\mathbf{M}$ of real square-integrable functions on $S^{1}$ (unitarized by multiplication by the square-root of the Jacobian). 
Let $R$ denote the defining representation of $G$, as a real 4-dimensional representation. Let $S$ denote the $G$-invariant real symmetric form

$$
S(\phi, \psi)=\int_{S^{1}} \operatorname{Re}\left(\phi_{1}(x) \bar{\psi}_{1}(x)-\phi_{2}(x) \bar{\psi}_{2}(x)\right) d x .
$$

With $\mathbf{L}=\mathbf{M} \times \mathbf{R}$, and $U=V \times R$, the context of Theorem 1 is exemplified, with however $U$ defined on the entire group $G$ rather than only on a one-parameter subgroup. However, there is a vacuum for $\mathbf{C}(\mathbf{L}, S)$ only with respect to the maximal compact subgroup $K$ of $G$; no other one-parameter subgroup admits a vacuum. The corresponding Hilbert space $\mathbf{H}$ is identifiable naturally with $L_{2}\left(S^{1}, C^{2}\right)$, with $U(t)$ acting by rotations on $S^{1}$ and as $e^{i t}$ on $C^{2}$.

$G$ as a whole acts continuously but non-unitarily on $\mathbf{H}$, and as a non*-preserving group of automorphisms of $\mathbf{C}$. In particular it transforms the vacuum into a linear functional with certain positivity properties (an infinite-dimensional analog of the finite-dimensional linear functional of the form $X \rightarrow \operatorname{tr} X D$, where $D$ is a similarity transform of a positive hermitian matrix of unit trace). This may be interpreted as an unstable particle production process.

In the case of the group $G=\mathrm{SU}(2,2)$ acting similarly on $U(2)$ by linear fractional transformations, with $R$ the real 8-dimensional form of the defining representation of $\operatorname{SU}(2,2)$, the situation is similar. The vacuum is then invariant under the maximal compact (and maximal) subgroup $K$ of $\mathrm{SU}(2,2) ; K$ is in fact the isometry group of $U(2)$ in its invariant metric, and identifiable locally with the isometry group of the Einstein Universe, which is identifiable with the universal cover of $U(2)$. Cf. esp. [8]. The non-invariance of the vacuum under all of $\mathrm{SU}(2,2)$ is expected in the case of a non-compact simple Lie group such as $\operatorname{SU}(2,2)$, and represents in physical terms a "broken symmetry" playing an essential role in a proposed comprehensive model [11].

The non-invariance of the vacuum also gives rise formally to a cocycle on the whole symmetry group, via normalization of the vacuum expectation values of the formal implementing operators. Proposals for the use of analogous cocycles, related to so-called "Schwinger terms", have been made by Faddeev [5] and others in the mathematical physical literature. These raise the question of the effective and rigorous extension of the action of the group in $\mathbf{K}$ by the introduction of appropriate generalized vectors.

5. The bosonic vacuum. The absence of a Hilbert space adjunction operation is intrinsic to the treatment of the Clifford algebra with 
an indefinite symmetric bilinear form, in a way that differs from the situation for bosons. The existence of an adjunction operation can then be regarded as implicit in the conventional assumption that the Weyl operators are unitary. There is however no compelling necessity for the universal unitarity of Weyl systems in quantum mechanics. Such unitarity is often argued on the basis of stability, but metastable systems are commonplace in quantum physics, and can arguably be associated with non-unitarity. Moreover, even in one dimension, there exist regular Weyl systems that are non-unitary and not similar to any unitary Weyl system (this observation is due to U. Mosco).

It will facilitate and clarify the treatment to introduce an algebraic Weyl algebra $W$ over a given Hilbertizable linear symplectic space $(\mathbf{L}, A)$. Relative to any given not necessarily unitary Weyl system $(\mathbf{K}, W)$ over $(\mathbf{L}, A)$, the set of all finite linear combinations of the $W(z), z \in \mathbf{L}$, forms an algebra $\mathbf{W}_{0}$; and if $\left(\mathbf{K}^{\prime}, W^{\prime}\right)$ is another such Weyl system, there is a unique algebraic isomorphism of $\mathbf{W}_{0}$ onto $\mathbf{W}_{0}^{\prime}$ that carries $W(z)$ into $W^{\prime}(z)$, for all $z \in \mathbf{L}$. The algebraic Weyl alge$b r a$ is defined as the equivalence class of all such algebras $\mathbf{W}_{0}$, within algebraic isomorphisms of the type indicated.

TheORem 2. Let $(\mathbf{L}, A)$ be a Hilbertizable linear symplectic space, and let $U(\cdot)$ be a continuous one-parameter group of linear symplectic transformations fixing no nonzero vector in $\mathbf{L}$. Suppose also that $A(U(t) x, y)$ is bounded as a function of $t \in R^{1}$ for fixed $x, y$ in $\mathbf{L}$.

Let $E$ denote a linear functional on the algebraic Weyl algebra over (L, A) with the properties: (i) $E(1)=1$; (ii) $E(W(-z))=\bar{E}(W(z))$; (iii) if $Q \rightarrow Q^{t}$ denotes the automorphism of $\mathbf{W}$ that carries $W(z)$ into $W(U(t) z)$, then for all $Q, R \in \mathbf{W}, E\left(Q^{t} R\right)$ is as a function of the boundary values of a bounded analytic function in the upper half-plane; (iv) $\phi(z)=E(W(z))$ is continuous as a function of $z$, and for fixed $z, \phi(s z)=o\left(e^{k s^{2}}\right)$ for arbitrarily small $k>0$, as $s \rightarrow \infty$.

Then there exists a complex Hilbert structure on $\mathbf{L}$, whose real Hilbertizable structure is the same as that given in $\mathbf{L}$ such that

(1) $U(\cdot)$ is unitary with positive self-adjoint generator on $\mathbf{H}$;

(2) $A(x, y)=\operatorname{Im}\langle x, y\rangle$;

(3) $E$ coincides with the free vacuum over $\mathbf{H}$.

REMARK. The condition that $A(U(t) x, y)$ be bounded for fixed $x, y$ is essentially implied by the other assumptions in case $E$ can be naturally extended to polynomials in the "fields" $\Phi(x)$, i.e. the generators 
of the one-parameter groups $\left[W(t x): t \in R^{1}\right]$, using the observation that the commutator of these fields is substantially the given form $A$. This property of $E$, in the nature of the existence of moments of the pseudo-distribution (in the probability sense) defined by $E$ (indeed the existence of first and second moments suffices) is often assumed in considerations involving the vacuum of a quantized field.

LEMMA. Irrespective of the assumptions on $E, \mathbf{L}$ admits a complex Hilbert space structure, to be denoted as $\mathbf{H}^{\prime}$, whose real Hilbertizable structure is the same as the given one in $\mathbf{L}$, relative to which the $U(t)$ are unitary, and such that $A(x, y)=\operatorname{Im}\langle x, y\rangle$ for arbitrary $x, y \in \mathbf{L}$.

Proof of lemma. As in the proof of the analogous lemma for Theorem 1 , there exists a $U(\cdot)$-invariant positive definite real symmetric form $S$ on $\mathbf{L}$ defining the given Hilbertizable structure. By the Hilbertizability of $(\mathbf{L}, A)$, there exists a unique bounded invertible real-linear operator $R$ on $\mathbf{L}$ such that $A(x, y)=S(R x, y)$ for all $x, y \in \mathbf{L}$; moreover, $R$ commutes with the $U(t)$, by virtue of its unicity and the invariance of $S$ and $A$. Since

$$
A(x, y)=S(R x, y)=-A(y, x)=-S(R y, x)=-S(x, R y),
$$

$R$ is skew-adjoint relative to the real Hilbert space with fundamental form $S$. Setting $J=-R\left(-R^{2}\right)^{-1 / 2}$, then $J$ is a bounded invertible linear operator on $\mathbf{L}$, commutative with the $U(t)$, and such that $J^{2}=$ -1 . Moreover, $A(J x, x)$ is non-negative and vanishes only if $x=0$, since

$$
A(J x, x)=S(R J x, x)=S\left(-R^{2}\left(-R^{2}\right)^{-1 / 2} x, x\right)=S\left(\left(-R^{2}\right)^{-1 / 2} x, x\right) .
$$

Accordingly the definition $\langle x, y\rangle^{\prime}=A(J x, y)+i A(x, y)$, together with $J$ as complex structure, endows $\mathbf{L}$ with the structure of a complex Hilbert space $\mathbf{H}^{\prime}$. Since the $U(t)$ commute with $J$ and leave $A$ invariant, they are unitary on $\mathbf{H}^{\prime}$.

Proof of theorem. Let $H$ denote the self-adjoint generator of the unitary group $U(\cdot)$ on $\mathbf{H}$ that exists by the lemma, and let $\mathbf{H}_{ \pm}$denote the subspaces on which $H$ is respectively positive and negative. According to the Weyl relations, $W(-z) W\left(z^{t}\right)=e^{i A\left(z^{t}, z\right) / 2} W\left(z^{t}-z\right)$. It follows that $e^{i A\left(z^{t}, z\right) / 2} E\left(W\left(z^{t}-z\right)\right)$ is as a function of $t$ in the class C of functions on $R^{1}$ that are continuous and are boundary values of bounded analytic functions in the upper half-plane. 
Now suppose $z \in \mathbf{H}_{-}$. Then $g(t)=e^{\left\langle z^{-t}, z\right\rangle}$ is in $\mathbf{C}$, and setting $f(t)=e^{i A\left(z^{t}, z\right) / 2} E\left(W\left(z^{t}-z\right)\right)$, then $f(t) g(t)$ is also in C. Now

$$
f(t) g(t)=e^{\operatorname{Re}\left\langle z^{t}, z\right\rangle / 2} E\left(W\left(z^{t}-z\right)\right)
$$

since

$$
\left\langle z^{-t}, z\right\rangle=\operatorname{Re}\left\langle z^{t}, z\right\rangle-i \operatorname{Im}\left\langle z^{t}, z\right\rangle .
$$

Replacing $z$ by $-z$, it follows that $e^{\operatorname{Re}\left\langle z^{t}, z\right\rangle / 2} E\left(W\left(-z^{t}+z\right)\right)$ is also in C, but this function of $t$ is the complex conjugate of $f(t) g(t)$, since $E(W(-z))=\bar{E}(W(z))$. It follows that $f(t) g(t)$ is a constant, and setting $t=0$, it results that $E\left(W\left(z^{t}-z\right)\right)=e^{\langle z, z\rangle / 2-\operatorname{Re}\left\langle z^{t}, z\right\rangle / 2}=$ $e^{\left\|z^{t}-z\right\|^{2} / 4}$; on replacing $z$ by $s z$, this grows too rapidly as a function of $s$, unless $z^{t}=z$, which is impossible for all $t$ unless $z=0$. Thus $\mathbf{H}$ consists only of the zero vector.

If $z \in \mathbf{H}_{+}$, then $h(t)=e^{-\left\langle z^{t}, z\right\rangle}$ is in $\mathbf{C}$, and

$$
f(t) h(t)=e^{-\operatorname{Re}\left\langle z^{t}, z\right\rangle} E\left(W\left(z^{t}-z\right)\right)
$$

is also in $\mathbf{C}$. The same argument as earlier shows that $f h$ is constant, as a function of $t$, and evaluating it by setting $t=0$, it results that $E\left(W\left(z^{t}-z\right)\right)=e^{-\left\|z^{t}-z\right\|^{2} / 4}$. Since vectors of the form $z^{t}-z$ are dense in $\mathbf{H}$ (the generator of $U(\cdot)$ is positive as a consequence of the absence of fixed nonzero vectors), it follows that $E(W(z))=e^{-\|z\|^{2} / 4}$ for all $z$ in $\mathbf{H}$. Thus $E$ coincides with the free vacuum expectation value functional for the boson field over $\mathbf{H}$.

6. Discussion. The question of unicity of the vacuum is readily treated by the methods used above. In the fermionic case, the complex structure is unique, but the remaining Hilbert space structure is unique only within a complex-linear transformation in $\mathrm{O}(\mathbf{L}, S)$. Such a transformation may be interpreted physically as that between different observers, whose vacua may thus appear distinct, although related by a well-defined symmetry, in the non-unitary case. In the non-unitary case the boson vacuum is unique [13].

Although the parallel between fermion and boson fields is strikingly close, there are fundamental differences both mathematically and physically. The antiparticle phenomenon appears inherent in the case of fermions, but some bosons such as photons appear as their own antiparticles. This seems reflected in the natural occurrence of indefinite forms, representing algebraic particle number, or a generalized charge, in the case of fermions, as in the Dirac theory. The murky 
treatment of antiparticles via "hole" theory, or the clearcut but opportunistic treatment via the interchange of creation and annihilation operators for the antifermions, may be regarded as in part precursors to the transition from an indefinite to a definite form deduced here via the constraint of stability for the vacuum.

For the stabilizable unitary representations of the conformal group $\mathrm{SU}(2,2)$, such as that for the massless Dirac equation, the vacuum gives rise to bounded holomorphic functions not only in the dual halfplane to the energy as above, but to such functions in the tube over an invariant convex cone in the dual of the Lie algebra (that of all infinitesimal conformal displacements into the future, in the case of the Dirac equation). In the section space of the spannor bundle, however, the massless Dirac equation characterizes non-trivial (indecomposable) quotients of $L_{2}$-representations, which cannot be separated from the other factors in the composition series, resulting in a fundamental overall vacuum that is only $K$-invariant, and so observerdependent. The choice of an observer at a point is interpretable as a choice of $K$, so that the isotropy subgroup implements changes of local frame of observation.

\section{REFERENCES}

[1] V. Bargmann and E. P. Wigner, Group theoretical description of relativistic wave equations, Proc. Natl. Acad. Sci. USA, 34 (1948), 211-233.

[2] R. J. Blattner, Automorphic group representations, Pacific J. Math., 8 (1958), 665-677.

[3] P. J. M. Bongaarts, The electron-positron field, coupled to external electromagnetic potentials, as an elementary $C^{*}$-algebra theory, Ann. Phys., 56 (1970), 108-139.

[4] G. G. Emch, Algebraic Methods in Statistical Mechanics and Quantum Field Theory, Wiley, New York, 1972.

[5] L. D. Faddeev, Operator anomaly for the Gauss law, Physics Lett., 145B (1984), 81-84.

[6] U. Haagerup, Solution of the similarity problem for cyclic representations of $C^{*}$-algebras, Ann. of Math., 118 (1983), 215-240.

[7] G. W. Mackey, Unitary representations of the Poincaré group, Appendix to 'Mathematical problems of relativistic physics', by I. E. Segal, Amer. Math. Soc., Providence, 1963.

[8] S. M. Paneitz, I. E. Segal, and D. A. Vogan, Jr., Analysis in space-time bundles, IV. Natural bundles deforming into and composed of the same invariant factors as the spin and form bundles, J. Funct. Anal., 75 (1987), 1-57.

[9A] B. Ørsted and I. E. Segal, A pilot model in two dimensions for conformally invariant particle theory, J. Funct. Anal. in press. 
[9] I. Segal, Mathematical characterization of the physical vacuum for linear BoseEinstein fields, Canad. J. Math., 13 (1961), 1-18.

[10] _ Algebraic quantization of induced bundles, in Physique quantique et geometrie, ed. D. Bernard \& Y. Choquet-Bruhat, Hermann, Paris, 1988, 119139.

[11] I. E. Segal, B. Ørsted, S. M. Paneitz, and D. A. Vogan, Jr., Explanation of parity nonconservation, Proc. Natl. Acad. Sci., 84 (1987), 319-323.

[12] V. S. Varadhan, Geometry of Quantum Theory, Van Nostrand, Princeton, $1968 / 70$.

[13] M. Weinless, Existence and uniqueness of the vacuum for linear quantized fields, J. Funct. Anal., 4 (1969), 350-379.

Received March 29, 1988. Research supported in part by NSF.

Massachusetts Institute of TeChNology

CAMBRIDGE, MA 02139 



\section{PACIFIC JOURNAL OF MATHEMATICS EDITORS}

\author{
V. S. VARADARAJAN \\ (Managing Editor) \\ University of California \\ Los Angeles, CA 90024 \\ Herbert Clemens \\ University of Utah \\ Salt Lake City, UT 84112 \\ THOMAS ENRIGHT \\ University of California, San Diego \\ La Jolla, CA 92093
}

R. FINN

Stanford University

Stanford, CA 94305

HeRmann FlaschKa

University of Arizona

Tucson, AZ 85721

VAUGHAN F. R. Jones

University of California

Berkeley, CA 94720

STEVEN KeRCKHOFF

Stanford University

Stanford, CA 94305
ROBION KIRBY

University of California

Berkeley, CA 94720

C. C. MOORE

University of California

Berkeley, CA 94720

HAROLD STARK

University of California, San Diego

La Jolla, CA 92093

\section{ASSOCIATE EDITORS}
R. ARENS
E. F. BECKENBACH
B. H. NeUMANN
F. WolF
K. YoshidA (1906-1982)

\section{SUPPORTING INSTITUTIONS}

UNIVERSITY OF ARIZONA

UNIVERSITY OF BRITISH COLUMBIA

CALIFORNIA INSTITUTE OF TECHNOLOGY

UNIVERSITY OF CALIFORNIA

MONTANA STATE UNIVERSITY

UNIVERSITY OF NEVADA, RENO

NEW MEXICO STATE UNIVERSITY

OREGON STATE UNIVERSITY

\author{
UNIVERSITY OF OREGON \\ UNIVERSITY OF SOUTHERN CALIFORNIA \\ STANFORD UNIVERSITY \\ UNIVERSITY OF HAWAII \\ UNIVERSITY OF TOKYO \\ UNIVERSITY OF UTAH \\ WASHINGTON STATE UNIVERSITY \\ UNIVERSITY OF WASHINGTON
}

The Supporting Institutions listed above contribute to the cost of publication of this Journal, but they are not owners or publishers and have no responsibility for its content or policies.

Mathematical papers intended for publication in the Pacific Journal of Mathematics should be in typed form or offset-reproduced (not dittoed), double spaced with large margins. Please do not use built up fractions in the text of the manuscript. However, you may use them in the displayed equations. Underline Greek letters in red, German in green, and script in blue. The first paragraph must be capable of being used separately as a synopsis of the entire paper. In particular it should contain no bibliographic references. Please propose a heading for the odd numbered pages of less than 35 characters. Manuscripts, in triplicate, may be sent to any one of the editors. Please classify according to the scheme of Math. Reviews, Index to Vol. 39. Supply name and address of author to whom proofs should be sent. All other communications should be addressed to the managing editor, or Elaine Barth, University of California, Los Angeles, California 90024.

There are page-charges associated with articles appearing in the Pacific Journal of Mathematics. These charges are expected to be paid by the author's University, Government Agency or Company. If the author or authors do not have access to such Institutional support these charges are waived. Single authors will receive 50 free reprints; joint authors will receive a total of 100 free reprints. Additional copies may be obtained at cost in multiples of 50 .

The Pacific Journal of Mathematics is issued monthly as of January 1966. Regular subscription rate: $\$ 190.00$ a year (5 Vols., 10 issues). Special rate: $\$ 95.00$ a year to individual members of supporting institutions.

Subscriptions, orders for numbers issued in the last three calendar years, and changes of address should be sent to Pacific Journal of Mathematics, P.O. Box 969, Carmel Valley, CA 93924, U.S.A. Old back numbers obtainable from Kraus Periodicals Co., Route 100, Millwood, NY 10546.

The Pacific Journal of Mathematics at P.O. Box 969, Carmel Valley, CA 93924 (ISSN 0030-8730) publishes 5 volumes per year. Application to mail at Second-class postage rates is pending at Carmel Valley, California, and additional mailing offices. Postmaster: send address changes to Pacific Journal of Mathematics, P.O. Box 969, Carmel Valley, CA 93924.

\section{PUBLISHED BY PACIFIC JOURNAL OF MATHEMATICS, A NON-PROFIT CORPORATION}




\section{Pacific Journal of Mathematics}

Vol. 137, No. $2 \quad$ February, 1989

Alain Connes and E. J. Woods, Hyperfinite von Neumann algebras and Poisson boundaries of time dependent random walks ............ 225

R. Coquereaux and D. Kastler, Remarks on the differential envelopes of

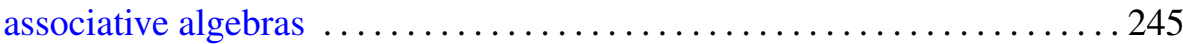

Uffe Haagerup, The injective factors of type $\mathrm{III}_{\lambda}, 0<\lambda<1 \ldots \ldots \ldots 265$

Vaughan Jones, On knot invariants related to some statistical mechanical

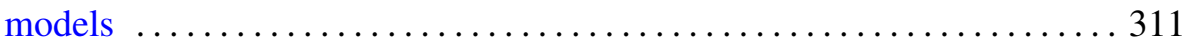

Paul Scott Muhly, Kichi-Suke Saito and Baruch Solel, Coordinates for triangular operator algebras. II ......................... 335

Klaus Schmidt, Mixing automorphisms of compact groups and a theorem by Kurt Mahler ........................................ 371

Irving E. Segal, Algebraic characterization of the vacuum for quantized

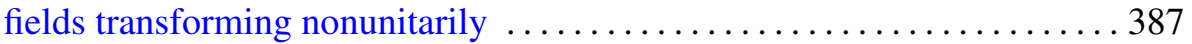

Colin Eric Sutherland and Masamichi Takesaki, Actions of discrete amenable groups on injective factors of type $\mathrm{III}_{\lambda}, \lambda \neq 1 \ldots \ldots \ldots \ldots 405$ 